\title{
High viscosity gas fluidization of fine particles: An extended window of quasihomogeneous flow
}

\author{
Jose Manuel Valverde and Antonio Castellanos \\ Facultad de Fisica, Universidad de Sevilla, Avenida Reina Mercedes s/n, 41012 Sevilla, Spain
}

(Received 21 April 2006; published 8 August 2006)

\begin{abstract}
We explore the role of gas viscosity in the behavior of gas-fluidized beds of fine powders by means of experimental measurements using nitrogen and neon as fluidizing gases, and theoretical considerations. The existence of a nonbubbling fluidlike regime has been recently observed in beds of fine powders fluidized with nitrogen. Our experiments with neon reveal a discontinuous transition from heterogeneous fluidization to a highly expanded homogeneous fluidization state. We point out that increasing gas viscosity enhances the coherence of agglomerate swarms, which promotes a local void-splitting mechanism, thus improving the uniformity of fluidization. Our theoretical analysis predicts that further increase of gas viscosity would produce a full suppression of the bubbling regime, i.e., the uniformly fluidized bed would undergo a direct transition to a turbulent regime as seen in beds of nanoparticles fluidized by nitrogen and in liquid-fluidized beds of moderate-density beads.
\end{abstract}

DOI: 10.1103/PhysRevE.74.021302

PACS number(s): 83.80.Fg, 47.55.Kf, 81.20.Ev

\section{INTRODUCTION}

Newly developed powder production and synthesis methods have intensified the role of fine powders in powder-based innovations where greater reactivity is desired. One of the best techniques available for industrial applications is fluidization which provides a high fluid-solid contact and reaction efficiency. As far as fluidization of "large" noncohesive particles (particle size $d_{p}>\sim 50 \mu \mathrm{m}$ ) is concerned, it has been traditionally observed that liquid-fluidized beds do not bubble, whereas visible fluid bubbles appear in gas-fluidized beds as soon as the gas velocity $v_{g}$ surpasses the minimum fluidization velocity [1]. The earliest attempt to delineate the transition between uniform and bubbling fluidization was based on a Froude number criterion that $\mathrm{Fr}=v_{g}^{2} /\left(g d_{p}\right)>1$ where $g$ is the gravitational acceleration for bubbling behavior. The Froude number is, however, too rough to offer a criterion for the transition from homogeneous to bubbling fluidization. One order of magnitude of difference has been found between, for example, air-fluidized glass beads ( $\mathrm{Fr}$ $\simeq 1.1)$ and air-fluidized lead shot $(\mathrm{Fr} \simeq 85) \quad[1]$. In addition, short-lived bubblelike voids have been observed in liquidfluidized beds of moderate-density beads $\left(\mathrm{Fr} \sim 10^{-3}-10^{-2}\right)$ and stable bubblelike voids have been seen to develop above a critical fluid velocity in liquid-fluidized beds of highdensity beads $\left(\mathrm{Fr} \sim 10^{-1}\right)[1,2]$. In order to estimate the largest stable size $D_{b}$ of isolated fluid bubbles, Harrison et al. [3] hypothesized that bubbles are no longer stable if their rising velocity $U_{b}$ exceeds the terminal velocity of the particles $v_{p 0} \simeq(1 / 18)\left(\rho_{p}-\rho_{f}\right) d_{p}^{2} g / \mu$, where $\rho_{p}$ is the particle density, $\rho_{f}$ is the fluid density, $\mu$ is the fluid viscosity, and we have neglected fluid inertia. Using the well-established semiempirical equation $U_{b} \simeq 0.7 \sqrt{g D_{b}}$ [4], they found that $D_{b} / d_{p}>\sim 10$ for bubbling beds and $D_{b} / d_{p}<\sim 1$ for uniformly fluidized beds.

Industry demands have shifted in recent years; a big part of the research focuses on gas fluidization of particles smaller than $\sim 20 \mu \mathrm{m}$, for which interparticle attractive forces have a strong relevance $[5,6]$. Recent data on gasfluidized beds of fine and ultrafine particles have demon- strated that there exists a window of bed expansion where the bed exhibits a nonbubbling fluidlike regime $[7,8]$. Gasfluidized fine particles agglomerate, driven by the dynamic equilibrium between the interparticle attractive force $F_{0}$ and flow shear, which supports the particle weight in the gravity field [6]. From this balance, the equation $\mathrm{Bo}_{g} \sim \kappa^{D+2}$ has been recently derived [6], where $\mathrm{Bo}_{g}$ (granular Bond number) is the ratio of $F_{0}$ to particle weight, $\kappa$ is the ratio of agglomerate size to particle size, and $D$ is the fractal dimension of the agglomerate $(D=\ln N / \ln \kappa, N$ being the number of particles in the agglomerate). Remarkably, gas viscosity should not affect agglomerate size (although $F_{0}$ may increase by gas adsorption to the surface of the particles, this effect is negligible at ambient pressure [9]). Thus, the nonbubbling fluidization regime exhibited by agglomerated fine particles has been referred to as agglomerate particulate fluidization (APF). Experimental data [7] suggest that initiation of visible bubbling in fluidized beds of fine cohesive powders can be predicted by the general Wallis criterion that stable bubbles appear when the propagation velocity of a voidage disturbance $u_{\phi}$ equals the elastic wave velocity $u_{e}$ of the bed. For APF we have $u_{\phi} \simeq \kappa^{2} \phi v_{p 0} n\left(1-\phi \kappa^{3} / N\right)^{n-1}$, where $\phi$ is the particle volume fraction, $n \simeq 5.6$ in the small-Reynoldsnumber regime (typically $\operatorname{Re}<\sim 10^{-2}$ for fine particles), and $u_{e} \simeq\left(g d_{p} \phi \kappa^{4} / N\right)^{1 / 2}[7]$.

In this paper we investigate the role of gas viscosity on the APF regime of a fine powder. In the experimental part of the work nitrogen and neon will be used as fluidizing gases. Measurements of $\phi$ and the settling velocity of the fluidized bed as a function of $v_{g}$ are carried out to identify the fluidization regimes and to characterize particle agglomerates. Local measurements of the concentration of solids have also been performed that serve us to discuss the role of gas viscosity in bed expansion and transition to the bubbling regime. Finally, our measurements will be rationalized on the basis of the Harrison et al., Froude number, and Wallis criteria modified for APF. The discussion will drive us to predict full suppression of bubbling for fluidized beds of micrometer-sized particles in the limit of high viscosity gas 
as observed in beds of nanoparticles fluidized with nitrogen and beds of noncohesive large beads fluidized by liquids.

\section{EXPERIMENTAL SETUP}

Experiments have been carried out using a commercially available xerographic toner (Canon CLC700 cyan toner) consisting of polyester fine particles $\left(d_{p} \simeq 8.3 \mu \mathrm{m}, \rho_{p}\right.$ $\simeq 1.2 \mathrm{~g} / \mathrm{cm}^{3}$ ). The fluidized bed consists of a vertical 4.42 -cm-diam vessel containing the powder which is subjected to a controlled flow of gas injected through a gas distributor at the bottom of the bed. We use nitrogen $\left(\mu_{\mathrm{N}_{2}}=1.79\right.$ $\left.\times 10^{-5} \mathrm{~Pa} \mathrm{~s}, \quad v_{p 0}=v_{\mathrm{N}_{2}}=2.5 \mathrm{~mm} / \mathrm{s}\right)$ and neon $\left(\mu_{\mathrm{Ne}}=3.21\right.$ $\times 10^{-5} \mathrm{~Pa} \mathrm{~s}, v_{p 0}=v_{\mathrm{Ne}}=1.4 \mathrm{~mm} / \mathrm{s}$ ) as fluidizing gases at ambient conditions. In all the measurements the powder bed is first driven into the bubbling regime by imposing a sufficiently high gas flow. In the bubbling regime the powder loses memory of previous processes [10]. Then the gas flow is decreased to the desired value and measurements are taken once the system has reached a reproducible stationary state. Both global and local measurements are performed to explore the role of gas viscosity. The particle volume fraction of the fluidized bed $\phi$ is derived from the height of the bed, which is measured by an ultrasonic sensor placed on top of the vessel. This device can determine distance with an accuracy of $\pm 0.01 \mathrm{~cm}$ (much smaller than local fluctuations in bed height) by sending an ultrasonic wave and measuring the time of reflection from the target.

The local concentration of solids is monitored from the backscattered light intensity (reflectance $R$ ) measured by an optical probe as it is described in Ref. [11]. The optics system consisted of a fiber optic reflection probe coupled to a spectrometer and a tungsten-halogen light source. The probe is a tight bundle of seven optical fibers (a hexagonal array of six illumination fibers disposed around one read fiber $400 \mu \mathrm{m}$ in diameter) embedded in a stainless steel ferrule with a $30^{\circ}$ window to remove specular reflection effects. (Although unavoidable flow perturbation is introduced by the probe upstream we expect it to be negligible downstream because of its sharp V-shaped tip.) We inserted the probe vertically at $1 \mathrm{~cm}$ depth in the center of the fluidized bed, and the backscattered light intensity (named hereafter the reflectance $R$ ) at $\lambda=550 \mathrm{~nm}$ was acquired each $24 \mathrm{~ms}$. According to our measurements, fluctuations in $R$ can be directly related to fluctuations in the local density. The characteristic frequency of fluctuations in the pressure due to gas bubbles in most bubbling beds is of the order of $1-5 \mathrm{~Hz}$ [12], and therefore sampling at $1 / 24 \mathrm{~ms} \simeq 40 \mathrm{~Hz}$ would be sufficient to detect not only bubbles but also small gas pockets if present. Although the local probe used in these experiments is invasive, we expect that the perturbation introduced in the flow by the local probe is only significant upstream, as suggested by the similarity between our results and results from noninvasive local pressure measurements on a fluidized bed of glass beads [10].

Additionally, settling experiments have been performed, which consist of suddenly stopping the gas flow after the fluidized bed has reached a stationary condition and measuring the initial settling velocity of the bed using the ultrasonic
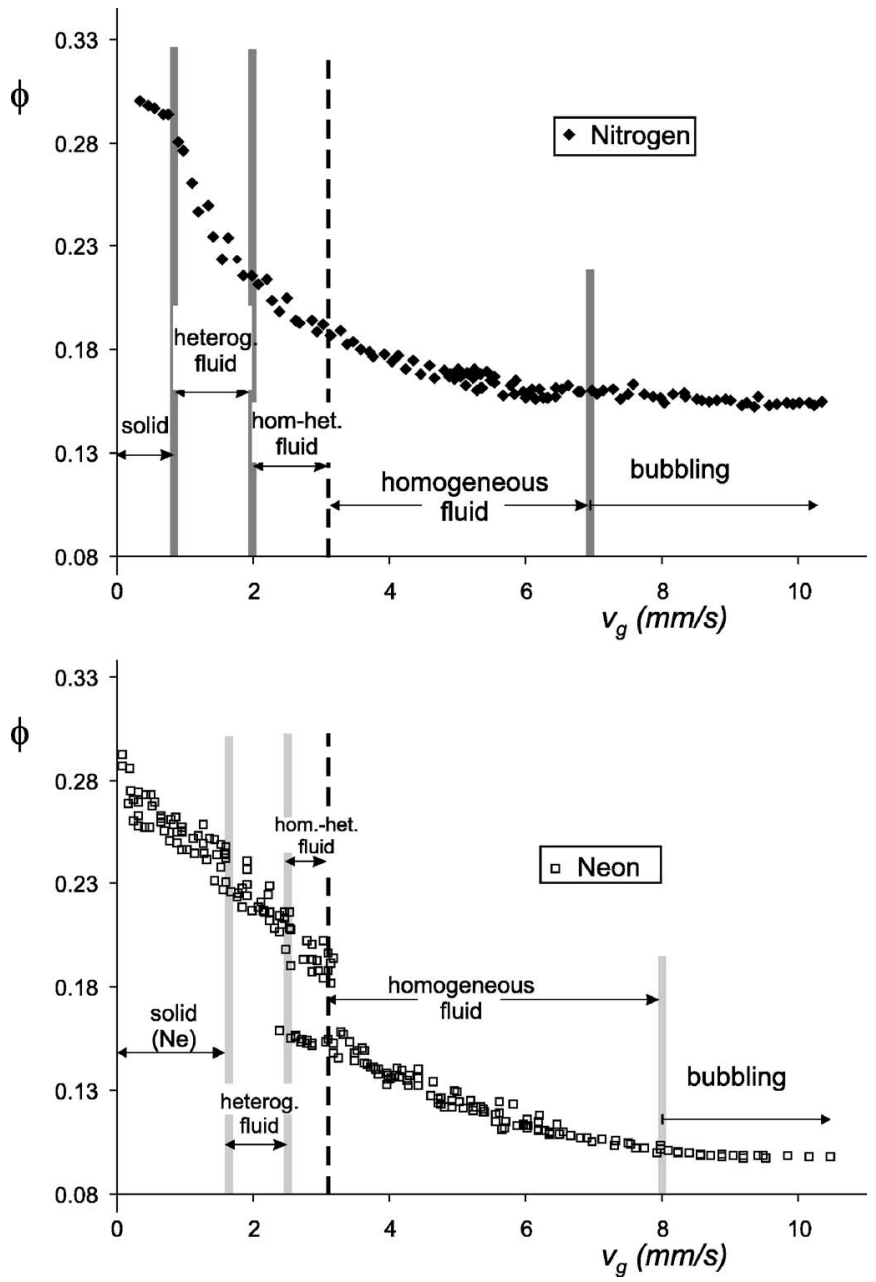

FIG. 1. Particle volume fraction of the fluidized bed $\phi$ vs gas velocity $v_{g}$ for fluidization with nitrogen (top) and neon (bottom). Fluidization regimes are delineated.

sensor as a function of the initial value of $\phi$. Settling experiments allow us to characterize particle agglomerates in the fluidized bed [6] in order to check whether gas viscosity plays a role. An extended report about the functioning of the fluidized bed apparatus can be found in Ref. [6].

\section{RESULTS AND ANALYSIS}

\section{A. Fluidization regimes}

Figure 1 displays data of the particle volume fraction of the fluidized bed $\phi$ vs gas velocity $v_{g}$ for fluidization with nitrogen (top) and neon (bottom). We show the flow regimes that have been identified according to the time signals measured of the local reflectance $R(t)$ [see typical examples of $R(t)$ in Fig. 2].

As was inferred in Ref. [13] from measurements of the tensile strength and effective diffusion, we observe that the fluidized bed transits to a solidlike regime (jamming transition) at $v_{g} \simeq 1 \mathrm{~mm} / \mathrm{s}$ for fluidization with nitrogen. We identify in this work the solidlike regime from the absence of fluctuations in the local reflectance time signal $R(t)$. On the other hand the jamming transition occurs at $v_{g} \simeq 1.8 \mathrm{~mm} / \mathrm{s}$ 


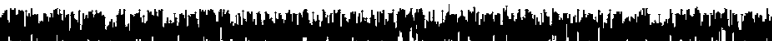

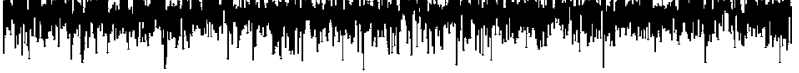

$R$

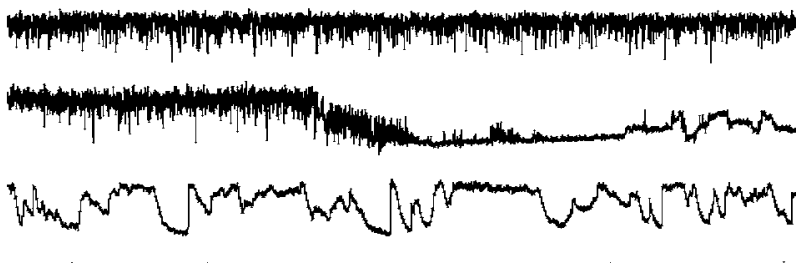

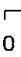

50

100

150

$t(s)$

FIG. 2. Typical time series of the reflectance (in a.u.) for different fluidization regimes. From top to bottom: bubbling fluidization, homogeneous fluidization, homogeneous-to-heterogeneous fluidization transitional regime, heterogeneous fluidization, and solidlike fluidization. In the examples the fluidizing gas is neon. Examples of reflectance time signals for fluidization with nitrogen can be seen in Ref. [11].

for fluidization with neon. Thus while in the interval $v_{g}$ $\in(1,1.8) \mathrm{mm} / \mathrm{s}$ the bed fluidized with neon remains in a solidlike state, fluidization becomes heterogeneous when it is fluidized with nitrogen. This heterogeneous state is characterized by the presence of long-lived solid network structures coexisting with transient craters and volcanoes. A footprint of such flow structures is clearly seen in $R(t)$, where time periods of low reflectance, due to the opening of craters, alternate with time periods of high reflectance and small fluctuations characteristic of the solidlike behavior. In the window $v_{g} \in(1.8,2.5) \mathrm{mm} / \mathrm{s}$ fluidization becomes heterogeneous for both gases and the data of $\phi$ vs $v_{g}$ are, within the experimental scatter, in the same curve (see Fig. 1). Between 2 and $3 \mathrm{~mm} / \mathrm{s}$, fluidization with nitrogen turns gradually from heterogeneous to homogeneous: the standard deviation of $R(t)$ decreases markedly [Fig. 3(a)] and the magnitude of fluctuations in the bed height declines gradually as $v_{g}$ is increased. In the interval between 2.5 and $3 \mathrm{~mm} / \mathrm{s}$ a novel phenomenon is observed when the powder is fluidized with neon. The fluidized bed in this interval cannot reach a stationary state, flipping back and forth between heterogeneous ( $\phi \simeq 0.15)$ and homogeneous fluidization $(\phi \simeq 0.15$, see Fig. $1)$. For $3 \mathrm{~mm} / \mathrm{s}<v_{g}<v_{m b}$, where $v_{m b}$ is the gas velocity at which the bed bubbles visibly, fluidization is homogeneous (bed height fluctuations and reflectance fluctuations are small) and the bed height decreases smoothly as $v_{g}$ is decreased.

\section{B. Settling experiments and particle agglomeration}

In Fig. 4 we show the data of the initial settling velocity of the top free surface of the fluidized bed $v_{s}$ vs $\phi$. Figure 5 is a plot of $v_{s}$ vs $v_{g}$, where it is seen, as expected, that $v_{s}$ $\simeq v_{g}$ in the homogeneous fluidization regime, whereas $v_{s}$ $<v_{g}$ in the bubbling fluidization regime. As reported in other works [6], $v_{s}$ can be well described by a modified Richardson-Zaki (RZ) law $v_{s}=v^{*}\left(1-\phi^{*}\right)^{n}$, where particle agglomerates are considered as effective spheres of size $d^{*}$. Here $v^{*}=v_{p 0} N / \kappa$ is the settling velocity of an individual agglomerate, $\phi^{*}=\phi \kappa^{3} / N$ is the volume fraction of the agglomerates in the fluidized bed, and $n \simeq 5$.6. The best fits of the

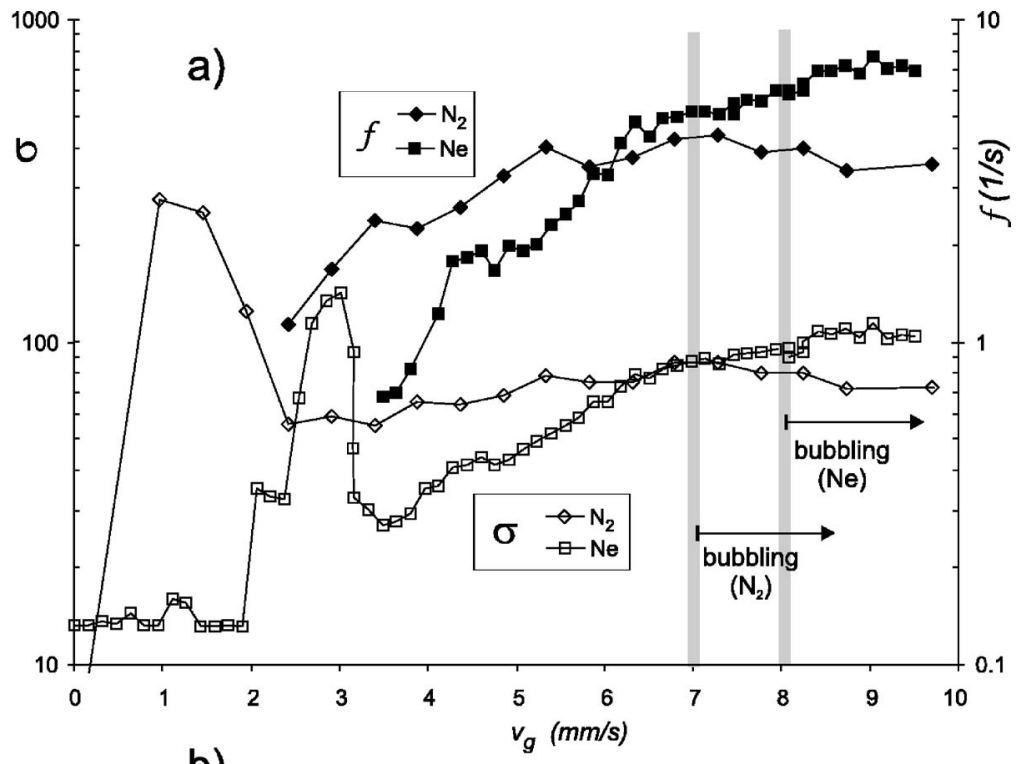

b)

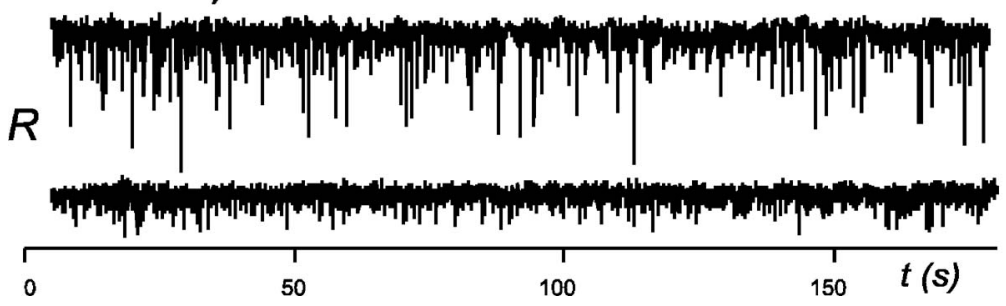

FIG. 3. (a) Standard deviation $\sigma$ (left axis) and average number of reflectance drops per unit time $f$ (right axis) vs gas velocity for nitrogen and neon fluidization. (b) Local reflectance in the nitrogen-fluidized bed (top) and neon-fluidized bed (bottom) for $v_{g}=4 \mathrm{~mm} / \mathrm{s}$. 


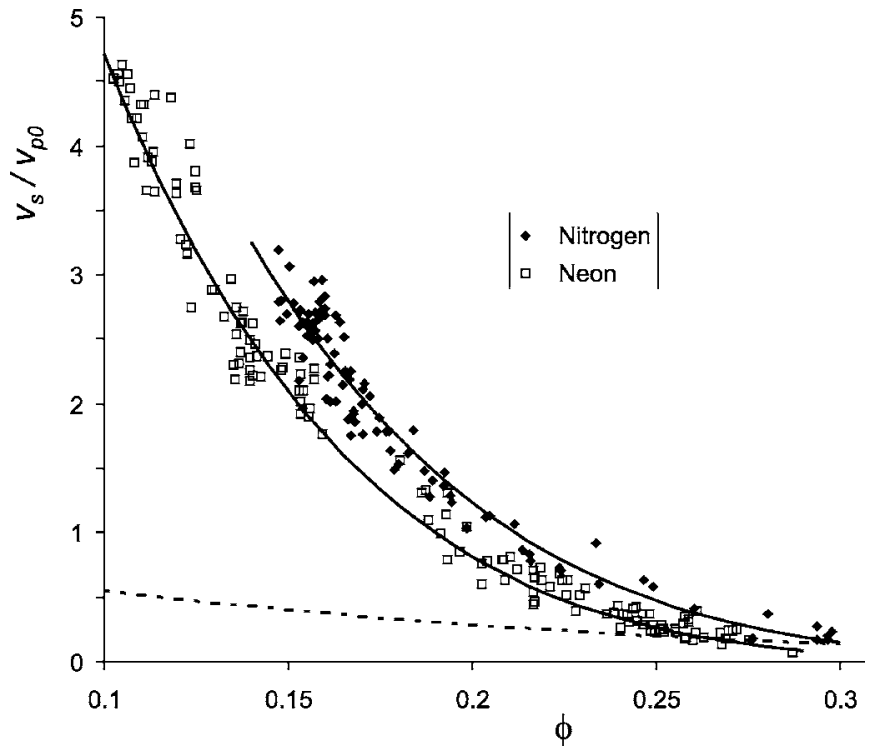

FIG. 4. Settling velocity $v_{s}$ of the fluidized bed (relative to the settling velocity of an individual particle) vs particle volume fraction for nitrogen and neon fluidization. The solid lines are the best fits curves of the modified RZ law to the data. The dashed line is the $\mathrm{RZ}$ prediction for individual particles.

modified RZ law to the data yield $N=114.7, \kappa=6.05$ (fluidization with nitrogen), and $N=109.8, \kappa=6.15$ (fluidization with neon). We have not observed clear differences between agglomerates taken out from the bed and visualized at the optical microscope (following the procedure described in Ref. [6]). Thus we cannot appreciate a relevant distinction between the agglomerates fluidized with different gases as theoretically predicted. Using these values and the theoretical equation $\mathrm{Bo}_{g} \sim \kappa^{D+2}$ we estimate $F_{0} \sim 10 \mathrm{nN}$ for the interparticle attractive force in the fluidized bed. Since we use dry gas and particles are essentially uncharged [14], the attractive force between the fluidized particles should arise mainly from the van der Waals interaction $F_{0} \simeq A d_{a} /\left(24 z_{0}^{2}\right)$, where $z_{0} \simeq 3-4 \AA$ is the distance of closest approach between two molecules, $A$ is the Hamaker constant, and $d_{a}$ is the typical size of the surface asperities [15]. Typically $A$ $\sim 10^{-19} \mathrm{~J}$ and $d_{a} \sim 0.2 \mu \mathrm{m}[15]$, which gives the same order

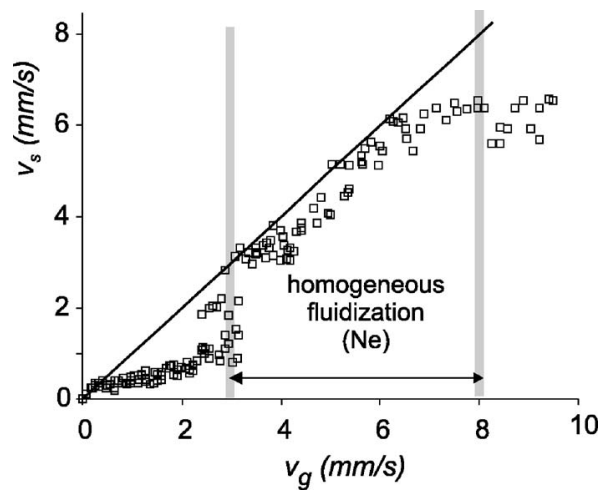

FIG. 5. Settling velocity $v_{s}$ of the fluidized bed vs gas velocity $v_{g}$ for fluidization with neon. The solid line represent the curve $v_{s}=v_{g}$.

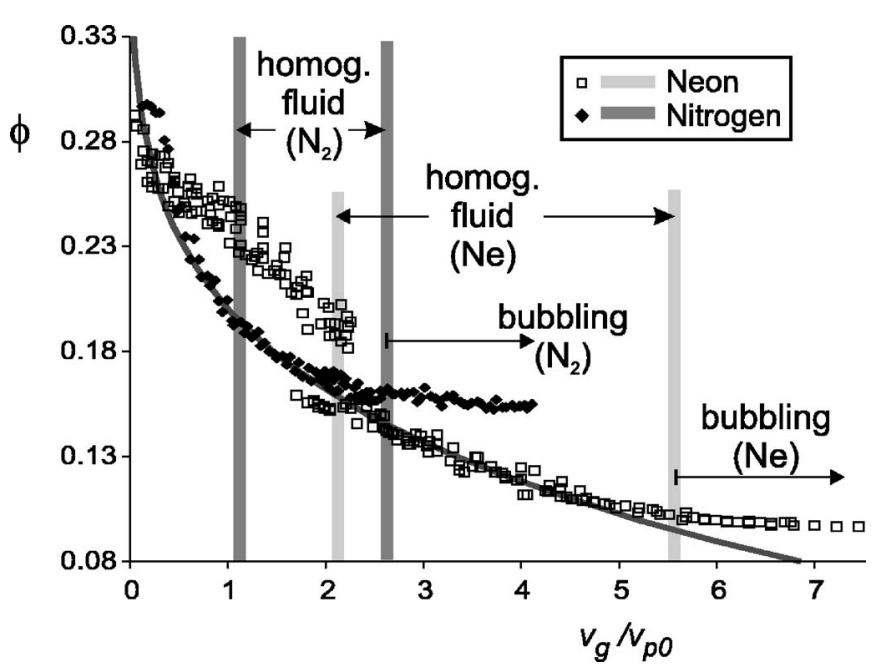

FIG. 6. Particle volume fraction of the fluidized bed $\phi$ vs the ratio of gas velocity $v_{g}$ to the terminal settling velocity of an individual particle $v_{p 0}$. The solid curve represents the modified RZ law.

of magnitude for $F_{0}$ as the one inferred from our experimental results.

In Fig. 6 we plot data of the particle volume fraction of the fluidized bed $\phi$ vs the ratio of gas velocity to terminal settling velocity of an individual particle, $v_{g} / v_{p 0}$. We also show the modified RZ equation for homogeneous fluidization, $v_{g} / v_{p 0} \simeq v_{s} / v_{p 0}=(N / \kappa)\left(1-\phi \kappa^{3} / N\right)^{n}$, where we have used the average values $N \simeq 112.3$ and $\kappa \simeq 6.1$. As expected the same law fits the data in the homogeneous regime of fluidization with both gases. Thus the discontinuous jump of $\phi$ for fluidization with neon at the heterogeneous-tohomogeneous fluidization transition $\left(v_{g} \simeq 3 \mathrm{~mm} / \mathrm{s}\right)$ can be quantified just from the relative change of viscosity using the RZ law: $\Delta \phi \simeq \kappa^{D-3}\left[\kappa^{1-D} v_{g} / v_{N_{2}}\right]^{1 / n}\left[\left(\mu_{\mathrm{Ne}} / \mu_{\mathrm{N}_{2}}\right)^{1 / n}-1\right] \simeq 0.03$. This result suggests that $\Delta \phi$ could be predicted from the RZ law for other gases, and would be larger the higher the gas viscosity.

\section{The local state}

Time signals of the local reflectance $R(t)$ of the fluidized bed in the homogeneous fluidization regimes are qualitatively similar for fluidization with both gases [see examples of $R(t)$ in Fig. 2 for fluidization with neon and Ref. [11] for examples of fluidization with nitrogen]. The main feature is the sudden drops in $R$ due to passage near the optical probe of gas pockets that do not develop into stable bubbles [11], in close analogy with observations in liquid-fluidized beds [2]. We observe, however, larger drops for nitrogen-fluidization in the vicinity of the transition to homogeneous fluidization [see Fig. 3(b)].

In Ref. [11] we presented an algorithm to calculate the average number of local gas pockets detected per unit time $f$. Results from this analysis are plotted in Fig. 3 along with the standard deviation $\sigma$ of $R(t)$, which is mainly determined by the size and frequency of the $R$ drops. Note that $f$ and $\sigma$ are clearly correlated. Near the heterogeneous fluidization regime $f_{\mathrm{Ne}}<f_{\mathrm{N}_{2}}$, yet the curves tend to converge as $v_{g}$ is in- 
creased. As the onset of nitrogen bubbling is approached we have $f_{\mathrm{Ne}} \simeq f_{\mathrm{N}_{2}}$ and $\sigma_{\mathrm{Ne}} \simeq \sigma_{\mathrm{N}_{2}}$. At the nitrogen-bubbling onset the coalescence of gas pockets dominates over the splitting process and $f_{\mathrm{N}_{2}}$ saturates. On the contrary $f_{\mathrm{Ne}}$ keeps growing and the neon-bubbling onset is further delayed.

The initiation of bubbling produces strong oscillations of the bed height due to bubbles burst on the free surface that are clearly distinguishable from the local surface eruptions produced by small gas pockets [11]. Incipient bubbling is also recognized by a curtail of bed expansion and by the clear deviation of the data from the RZ law $\left(v_{g}>v_{s}\right.$; se Fig. 5 ). We must note, however, that the transition to bubbling is not sharply defined. As seen in Figs. 1, 3, and 5 the system approaches the bubbling regime gradually in analogy with the behavior of liquid-fluidized beds of high density beads [2].

\section{DISCUSSION AND CONCLUSIONS}

The improvement of fluidization uniformity and delay of visible bubbling when we use neon as fluidizing gas must be a consequence of the enhancement of the splitting mechanism of gas pockets, reminding us of the efficiency of fluid pocket breakup observed in liquid-fluidized beds [2]. Since the resistance of the swarm of solids surrounding the gas pocket to be diluted should be higher the higher the gas viscosity, a possible explanation would be the increase of the coherence of the swarm of agglomerates that approaches the void and destabilizes it [15]. Coherent solid jets can make indentations more easily at gas pockets instead of flowing around it. In order to make some estimations of the largest stable size of a single gas pocket we will use the empirical criterion of Harrison et al. [3] $\left(U_{b} \simeq 0.7 \sqrt{g D_{b}} \sim v_{p 0}\right)$. For APF we must use the free-falling velocity of the agglomerate $v^{*}=v_{p 0} N / \kappa$ instead of $v_{p 0}$. Calculations yield $D_{b} \simeq 480 \mu \mathrm{m}$ $\sim 10 d^{*}$ (nitrogen fluidization) and $D_{b} \simeq 130 \mu \mathrm{m} \sim 3 d^{*}$ (neon fluidization). The breakup of single gas pockets in neon at smaller sizes explains why, far from bubbling, where the concentration of gas pockets is small and their collective interaction is negligible, we observe smaller drops in the reflectance [Fig. 3(b)]. This is reflected at the macroscopic level in the increased bed expansion for fluidization with neon. Moreover, due to the smaller size of local gas pockets in neon fluidization their critical concentration (or frequency) for coalescing into large bubbles is higher, thus allowing for further expansion of the bed and bubbling onset. The physical picture would consist of rising gas pockets rapidly split by coherent solid pockets before being able to coalesce into large bubbles. Consequently we have at the maximum expanded state $\phi=\phi_{b} \simeq 0.155 \pm 0.01$ and $f \simeq 4 \mathrm{~s}^{-1}$ for nitrogen, whereas $\phi=\phi_{b} \simeq 0.096 \pm 0.005$ and $f \simeq 8 \mathrm{~s}^{-1}$ for neon.

In Fig. 7 we plot the difference between the velocity of the elastic response of the bed $u_{e}$ and the velocity of a voidage disturbance $u_{\phi}$ as a function of $\phi[7]$ and for a range of values of the gas viscosity. It is seen that the bubbling condition $\left(u_{e}=u_{\phi}\right)$ is met for theoretical values of $\phi$ close to the experimental ones for fluidization with the two gases tested. It must be noted, however, that because the transition to bubbling is not sharp, there is a significant uncertainty in the

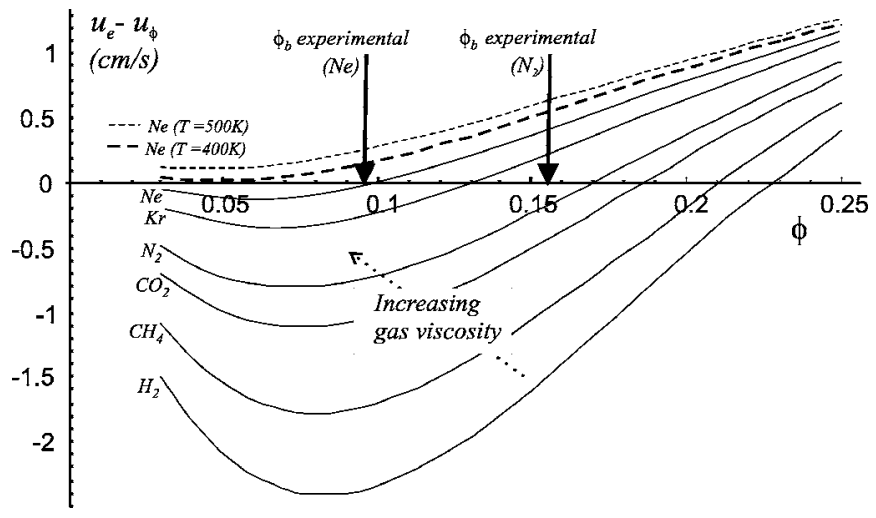

FIG. 7. Difference between the elastic wave velocity and the velocity of a voidage disturbance as a function of the particle volume fraction for fluidization of our powder system with different gases. $\mathrm{H}_{2}: \mu=0.9 \times 10^{-5} \mathrm{~Pa} \mathrm{~s} ; \mathrm{CH}_{4}: \mu=1.12 \times 10^{-5} \mathrm{~Pa} \mathrm{~s} ; \mathrm{CO}_{2}: \mu$ $=1.5 \times 10^{-5}$ Pa s; $\mathrm{N}_{2}: \mu=1.79 \times 10^{-5}$ Pa s; Kr: $\mu=2.56 \times 10^{-5}$ Pa s; $\mathrm{Ne}: \mu=3.21 \times 10^{-5} \mathrm{Pas}(T=300 \mathrm{~K})$. Ne: $\mu=3.89 \times 10^{-5} \mathrm{Pas}(T$ $=400 \mathrm{~K})$. Ne: $\mu=4.5 \times 10^{-5} \mathrm{~Pa} \mathrm{~s}(T=500 \mathrm{~K})$.

location of the experimentally measured values. Better agreement is obtained for fluidization with neon, which can be due to the larger size of bubbles for fluidization with nitrogen that produces higher bed height fluctuations and thus increases the uncertainty in the measurement of $\phi$ near the bubbling point as can be seen in Fig. 1.

Figure 7 predicts suppression of bubbling regime for $\mu>\sim 4 \times 10^{-5} \mathrm{~Pa}$ s. In this limit it would be expected that particle agglomerates experience a direct transition from uniform fluidization to elutriation, which is reminiscent of the behavior of moderate-density beads fluidized by liquid [2] and beds of nanoparticles fluidized with nitrogen [8]. On the other hand, Fig. 7 predicts that, for $\mu<\sim 1 \times 10^{-5}$ Pa s, bubbling $\left(u_{e}=u_{\phi}\right)$ would initiate shortly after the solid-to-fluid transition since $\phi_{b}>\sim 0.2$ (see Fig. 1). In the hypothetic case of $\mathrm{H}_{2}$ for example $\left(\mu_{\mathrm{H}_{2}} \simeq 0.9 \times 10^{-5} \mathrm{Pas}\right)$, we obtain from the Harrison criterion $D_{b} \simeq 2 \mathrm{~mm} \sim 40 d^{*}$, which also suggests a direct transition from heterogeneous to bubbling fluidization if this gas had been used. In an extended work we plan to check experimentally these results using a variety of gases at different temperatures.

To our knowledge we are the first workers to explore the effect of gas viscosity on the nonbubbling fluidization regime of fine powders. Yet it is worth noting that, in close analogy with results from our work, previous investigations on the effect of gas viscosity on the bubbling fluidization regime of coarser particles (typically $d_{p}>\sim 50 \mu \mathrm{m}$ ) indicate a decrease of the mean bubble size due to enhanced bubblesplitting mechanism for high viscosity gases $[15,16]$.

In conclusion, it has been shown that an increment of gas viscosity opens up a new window of highly expanded fluidization state for micrometer-sized particles. In the nonbubbling fluidization regime the bed expands according to the RZ law modified for particle agglomerates, whose size is not affected by gas viscosity as theoretically expected. Theoretical estimations and local measurements indicate that enhanced splitting of local gas pockets is favored in the case of a high viscosity gas possibly due to the increased resistance 
of coherent swarms of agglomerates to be diluted. Traditionally the behavior of noncohesive large particles fluidized by gas, exhibiting a direct transition from solid to bubbling behavior, has been considered as radically different from the behavior of liquid-fluidized beds, where nonbubbling fluidization is the common situation. In this work we have shown that moderate-density micrometer-sized particles fluidized by gas behave similarly to liquid-fluidized beds of high density beads, showing bubbling transition at high fluid velocities [2]. This analogy can be further illustrated by analyzing the Froude number $\mathrm{Fr}=v_{g}^{2} /\left(g d^{*}\right)$. In our system we have $\mathrm{Fr}$ $\simeq 1.8 \times 10^{-2}$ at the transition from heterogeneous to homogeneous fluidization and $\mathrm{Fr} \simeq 10^{-1}$ at the onset of bubbling (for fluidization with both gases) as found for liquidfluidized beds of dense particles at the development of transient and permanent bubbles, respectively [2]. From a continuum approach based on the Wallis criterion for bubbling onset and from the Harrison empirical criterion for the maximum stable size of bubbles we predict that the bed would transit directly from uniform fluidization to elutriation in the limit of high viscosity gas $\left(\mu>\sim 4 \times 10^{-5} \mathrm{~Pa} \mathrm{~s}\right)$ whereas the transition would be from heterogeneous fluidization to bubbling in the limit of small gas viscosity $(\mu<\sim 1$ $\left.\times 10^{-5} \mathrm{~Pa} \mathrm{~s}\right)$. Direct transition from uniform to turbulent fluidization has been observed in other systems such as gasfluidized beds of nanoparticles [8] and liquid-fluidized beds of moderate-density beads [2] (interestingly similar Froude numbers are reported for such apparently disparate systems, Fr $\left.\sim 10^{-3}-10^{-2}[1,8]\right)$. On the other hand, direct transition from solidlike to bubbling fluidization is commonly observed in gas-fluidized beds of noncohesive large particles. Gasfluidized beds of moderate-density micrometer-sized particles belong therefore to an intermediate class of systems in which a change of gas viscosity may be critical in shifting the balance to one or the other limiting behaviors.

We acknowledge Xerox Foundation and Spanish Ministerio de Ciencia y Tecnologia (Contract No. BMF2003-01739).
[1] G. M. Homsy, Appl. Sci. Res. 58, 251 (1998).

[2] P. Duru and E. Guazelli, J. Fluid Mech. 470, 359 (2002).

[3] D. Harrison et al., Trans. Inst. Chem. Eng. 39, 202 (1961).

[4] A. C. Hoffmann and J. G. Yates, Chem. Eng. Commun. 41, 133 (1986).

[5] J. P. K. Seville et al., Processing of Particulate Solids (Chapman and Hall, London, 1997).

[6] A. Castellanos, Adv. Phys. 54, 263 (2005).

[7] J. M. Valverde et al., Phys. Rev. E 67, 051305 (2003).

[8] C. Zhu et al., AIChE J. 51, 426 (2005).

[9] H. W. Piepers et al., Powder Technol. 37, 55 (1984).
[10] J. M. Valverde, A. Castellanos, and M. A. S. Quintanilla, Contemp. Phys. 44, 389 (2003).

[11] J. M. Valverde et al., Phys. Rev. E 67, 016303 (2003).

[12] F. Johnsson et al., Int. J. Multiphase Flow 26, 663 (2000).

[13] J. M. Valverde, A. Castellanos, and M. A. S. Quintanilla, Phys. Rev. Lett. 86, 3020 (2001).

[14] H. Krupp, Adv. Colloid Interface Sci. 1, 111 (1967).

[15] K. Rietema, The Dynamics of Fine Powders (Elsevier, London, 1991).

[16] S. Rapagna, P. U. Foscolo, and L. G. Gibilaro, Int. J. Multiphase Flow 20, 305 (1994). 